\title{
cmaJOPEN
}

\section{Virtual care use before and during the COVID-19 pandemic: a repeated cross-sectional study}

\author{
R. Sacha Bhatia MD MBA, Cherry Chu MSc, Andrea Pang MPH, Mina Tadrous PharmD PhD, \\ Vess Stamenova PhD, Peter Cram MD MBA
}

Abstract

Background: The coronavirus disease 2019 (COVID-19) pandemic is thought to have increased use of virtual care, but populationbased studies are lacking. We aimed to assess the uptake of virtual care during the COVID-19 pandemic using comprehensive population-based data from Ontario.

Methods: This was a repeated cross-sectional study design. We used administrative data to evaluate changes in in-person and virtual visits among all residents of Ontario before (2012-2019) and during (January-August 2020) the COVID-19 pandemic. We included all patients who had an ambulatory care visit in Ontario. We excluded claims for patients who were not Ontario residents or had an invalid or missing health card number. We compared monthly or quarterly virtual care use across age groups, neighbourhood income quintiles and chronic disease subgroups. We also examined physician characteristics that may have been associated with virtual care use.

Results: Among all residents of Ontario (population 14.6 million), virtual care increased from 1.6\% of total ambulatory visits in the second quarter of 2019 to $70.6 \%$ in the second quarter of 2020 . The proportion of physicians who provided 1 or more virtual visits per year increased from $7.0 \%$ in the second quarter of 2019 to $85.9 \%$ in the second quarter of 2020 . The proportion of Ontarians who had a virtual visit increased from $1.3 \%$ in 2019 to $29.2 \%$ in 2020 . Older patients were the highest users of virtual care. The proportion of total virtual visits that were provided to patients residing in rural areas (v. urban areas) declined significantly between 2012 and 2020 , reflecting a shift in virtual care to a service increasingly used in urban centres. The rates of virtual care use increased similarly across all conditions and across all income quintiles.

Interpretation: Our findings show that Ontario's approach to virtual care led to broad adoption across all provider groups, patient age, types of chronic diseases and neighborhood income. These findings have policy implications, including use of virtual care billing codes, for the ongoing use of virtual care during the second wave of the pandemic and beyond.

V irtual care, commonly defined as medical care delivered at a distance by means of technology, has existed since the 1970s. ${ }^{1}$ Despite substantial interest and investment in virtual care by both the federal and provincial governments, ${ }^{2-4}$ widespread adoption was modest. ${ }^{5,6}$ Research suggested that barriers to virtual care uptake in Ontario before the pandemic included limited physician reimbursement and government mandates that virtual visits use an approved video platform. ${ }^{7}$

As the novel coronavirus disease 2019 (COVID-19) pandemic evolved, payers around the globe acted with unprecedented speed by altering fee schedules to encourage virtual visits to reduce the risk of viral transmission, protect patients with chronic diseases who need ongoing medical attention and conserve personal protective equipment. ${ }^{8}$ In Ontario, during the first wave of the pandemic, the Ontario Health Insurance Plan quickly approved new temporary billing codes that allowed any type of technology, including telephone calls and commercial videoconferencing software (e.g., Skype, Zoom), to be used for virtual care. These temporary billing codes, as well as the preexisting video visit codes, are reimbursed at the same amount as in-person visits.

It has been widely reported that virtual care adoption has accelerated during the COVID-19 pandemic, but published data are limited. Previous studies have largely been limited to nongeneralizable patient subgroups. ${ }^{910}$ There has also been widespread concern that virtual care may worsen disparities because vulnerable populations (e.g., older adults, lower-income

\section{Competing interests: None declared.}

This article has been peer reviewed.

Correspondence to: Sacha Bhatia, sacha.bhatia@wchospital.ca CMAJ Open 2021. DOI:10.9778/cmajo.20200311 
patients) may lack proficiency in or access to the technology required for virtual care; empirical data to support this assertion are largely lacking.

We sought to quantify the uptake and use of virtual care for the full population of Ontario, Canada (population 14.6 million) before and during the COVID-19 pandemic and examine uptake among circumscribed vulnerable populations.

\section{Methods}

\section{Study design and setting}

We conducted a population-based repeated cross-sectional study of all ambulatory patient visits in Ontario beginning before the COVID-19 pandemic (Jan. 1, 2012) and extending to Aug. 31, 2020 (or until the end of the second quarter of 2020 for quarterly data). Before the pandemic, the only funded virtual care in Ontario was through the Ontario Telemedicine Network video visit platform, which allowed physicians who signed up for the service to bill for each visit on a fee-for-service basis. New temporary billing codes were introduced on Mar. 14, 2020 that expanded reimbursement to include telephone calls and Skype, Zoom and FaceTime sessions (Appendix 1, Supplemental Table S1, available at www. cmajopen.ca/content/9/1/E107/suppl/DC1); these codes have been continued indefinitely. The specifics of virtual visits, including modality and documentation, are left to the discretion of the physician. These codes apply to physicians but not physician assistants or nurse practitioners.

\section{Data sources}

We conducted this study using the Ontario Health Insurance Plan claims database (which records all health care services delivered by physicians to Ontario patients who are eligible for coverage) and the Registered Persons Database (which contains demographic information of all patients covered under the Ontario Health Insurance Plan). We used the Postal Code ${ }^{\mathrm{OM}}$ Conversion File ${ }^{11}$ to convert all patient postal codes to neighbourhood income quintiles. To determine subgroups of patients with chronic diseases, we used the Discharge Abstract Database (which records all inpatient hospital admissions), the National Ambulatory Care Reporting System (which contains data on all hospital- and community-based ambulatory care, including emergency department visits) and various ICES validated disease-specific registries. ICES is an independent, nonprofit research institute whose legal status under Ontario's health information privacy law allows it to collect and analyze health care and demographic data, without consent, for health system evaluation and improvement. Databases were linked by means of unique encoded identifiers and analyzed at ICES.

\section{Population}

We identified all ambulatory in-person visits and virtual care visits using relevant physician billing codes. We excluded claims for patients who were not Ontario residents or who had an invalid or missing health card number. We captured all eligible physician visits, irrespective of specialty.
We further identified patients with ambulatory-caresensitive conditions including chronic obstructive pulmonary disease, heart failure, asthma, hypertension and diabetes using existing ICES registries and previously published diseaseidentifying algorithms. ${ }^{12-16}$ We identified patients with serious mental illness by at least 2 outpatient claims or 1 inpatient claim with the corresponding International Classification of Diseases, 9th revision (ICD-9) or International Statistical Classification of Diseases and Related Health Problems, 10th revision (ICD-10) code for schizophrenia and psychotic disorders or bipolar disorder in the previous 12 months. ${ }^{17}$ We identified patients with angina by at least 1 emergency department visit with the relevant ICD-9 or ICD-10 code in the previous 12 months (Appendix 1, Supplemental Table S2).

\section{Statistical analysis}

For each quarter of our study period, we examined the number of in-person and virtual visits; the proportion of ambulatory visits that were virtual (v. in-person); the proportion of Ontario residents eligible for health care services who received at least 1 virtual visit; and the proportion of providers who billed for at least 1 virtual visit. We conducted $\chi^{2}$ tests to assess the sociodemographic characteristics (age category, sex, region of residence, neighbourhood income quintile and rurality) of patients who used virtual care across the years 2012, 2016 and 2020. We categorized each patient as residing in a rural or urban environment based on the postal code of residence using the Rurality Index for Ontario (RIO) score. The RIO was developed by the Ontario Ministry of Health and Long-Term Care as a method to measure fairly and consistently a community's degree of rurality based on its postal code. $^{18}$

We compared monthly or quarterly virtual care use across age groups (visits per 1000 people per unit of time), neighbourhood income quintiles (visits per 1000 people per unit of time) and chronic disease subgroups (visits per 100 people). For these subgroups, we calculated the monthly or quarterly percentage growth for June 2019 or the third quarter of 2019, and June 2020 or the second quarter of 2020, and compared these values using the Kruskal-Wallis test. We performed all analyses in SAS 9.4 (SAS Institute).

\section{Ethics approval}

Use of these databases for the purposes of this study was authorized under section 45 of Ontario's Personal Health Information Protection Act, which does not require review by a research ethics board.

\section{Results}

Table 1 shows the demographic characteristics of patients who had at least 1 virtual care visit in 2012, 2016 or 2020, and those who used no virtual care in 2020. Compared to patients who used virtual care during the prepandemic period (2012 and 2016), the proportion of adults aged 65 years or more who used virtual care in 2020 increased significantly $(19.4 \%$ v. $27.4 \%, p<0.001)$, as did the proportion of women 


\begin{tabular}{|c|c|c|c|c|c|c|}
\hline \multirow[b]{2}{*}{ Characteristic } & \multicolumn{3}{|c|}{ Received at least 1 virtual visit; no. (\%) of patients } & \multirow[b]{2}{*}{$p$ value } & \multirow{2}{*}{$\begin{array}{l}\text { Did not receive any } \\
\text { virtual visit in 2020; } \\
\text { no. (\%) of patients } \\
\quad n=3162395\end{array}$} & \multirow[b]{2}{*}{$p$ value } \\
\hline & $\begin{array}{c}2012 \\
n=51186\end{array}$ & $\begin{array}{c}2016 \\
n=146104\end{array}$ & $\begin{array}{c}2020 \\
n=4927830\end{array}$ & & & \\
\hline $18-49$ & $27028(52.8)$ & $74561(51.0)$ & $1822278(37.0)$ & \multicolumn{3}{|c|}{$1227511(38.8)$} \\
\hline $50-64$ & $10874(21.2)$ & $34954(23.9)$ & $1232489(25.0)$ & \multicolumn{3}{|c|}{$616658(19.5)$} \\
\hline $65-79$ & $7437(14.5)$ & $19956(13.7)$ & $992640(20.1)$ & \multicolumn{3}{|c|}{$378914(12.0)$} \\
\hline$\geq 80$ & $2468(4.8)$ & $6416(4.4)$ & $357062(7.2)$ & \multicolumn{3}{|c|}{$162431(5.1)$} \\
\hline \multicolumn{2}{|l|}{ Region } & & & \multicolumn{2}{|l|}{$<0.001$} & $<0.001$ \\
\hline Central & $4609(9.0)$ & $18403(12.6)$ & $1707781(34.7)$ & \multicolumn{3}{|c|}{$1053216(33.3)$} \\
\hline East & $17556(34.3)$ & $38709(26.5)$ & $1229707(25.0)$ & \multicolumn{3}{|c|}{$741447(23.4)$} \\
\hline North & $20389(39.8)$ & $45413(31.1)$ & $238786(4.8)$ & \multicolumn{3}{|c|}{$177070(5.6)$} \\
\hline Toronto & $1963(3.8)$ & $4337(3.0)$ & $454743(9.2)$ & \multicolumn{3}{|c|}{$266088(8.4)$} \\
\hline West & 6669 (13.0) & 39242 (26.9) & $1296813(26.3)$ & \multicolumn{3}{|c|}{$924574(29.2)$} \\
\hline \multicolumn{3}{|l|}{ Income quintile } & & \multicolumn{2}{|l|}{$<0.001$} & $<0.001$ \\
\hline 1 (lowest) & $13658(26.7)$ & $41111(28.1)$ & $943988(19.2)$ & \multicolumn{3}{|c|}{629119 (19.9) } \\
\hline 2 & $10182(19.9)$ & 30966 (21.2) & $974288(19.8)$ & \multicolumn{3}{|c|}{616595 (19.5) } \\
\hline Rural ( $\geq 40)$ & $14666(28.6)$ & 27145 (18.6) & 290401 (5.9) & \multicolumn{3}{|c|}{$204317(6.5)$} \\
\hline Missing & $3164(6.2)$ & $6238(4.3)$ & $32842(0.7)$ & & $33956(1.1)$ & \\
\hline
\end{tabular}

(49.4\% v. $56.6 \%, p<0.001)$. The proportion of total virtual visits that were provided to patients residing in rural areas (v. urban areas) declined significantly between 2012 and 2020 $(28.6 \%$ v. $5.9 \%, p<0.001)$, reflecting a shift in virtual care from a service used in Ontario's rural areas to a service increasingly used in urban centres.

Virtual care use increased with increasing age over the study period (Figure 1), which suggests that older adults increased their use of virtual visits along with younger adults. The rise in virtual care use from the prepandemic period to during the pandemic was both clinically and statistically similar across neighbourhood income quintiles (Figure 2). These results suggest that residents of lower-income neighbourhoods were not significantly disadvantaged.

Appendix 1, Supplemental Figure S1 shows the distribution of virtual visits in 2012, 2016 and 2020. The majority of patients (ranging from $81.3 \%$ to $88.7 \%$ ) had $1-5$ virtual visits per year at all 3 time periods; however, there was a significant increase in the number of patients with more than 5 virtual visits in 2020 compared to the earlier periods.

\section{Uptake of virtual care}

Figures $3 \mathrm{~A}$ and $3 \mathrm{~B}$ show longitudinal changes in in-person and virtual visits from Jan. 1, 2012, to June 30, 2020. During the prepandemic period, virtual care use increased slowly, from $0.2 \%$ of total ambulatory visits in the first quarter of 2012 to $1.8 \%$ of total ambulatory visits in the fourth quarter of 2019. With the onset of the pandemic, virtual visit volumes increased rapidly, reaching $70.2 \%$ of ambulatory visits during the second quarter of 2020 (Figure 3B). Almost threequarters $(73.7 \%)$ of all primary care visits and $63.9 \%$ of specialty care visits were delivered virtually in the second quarter 


\section{Research}

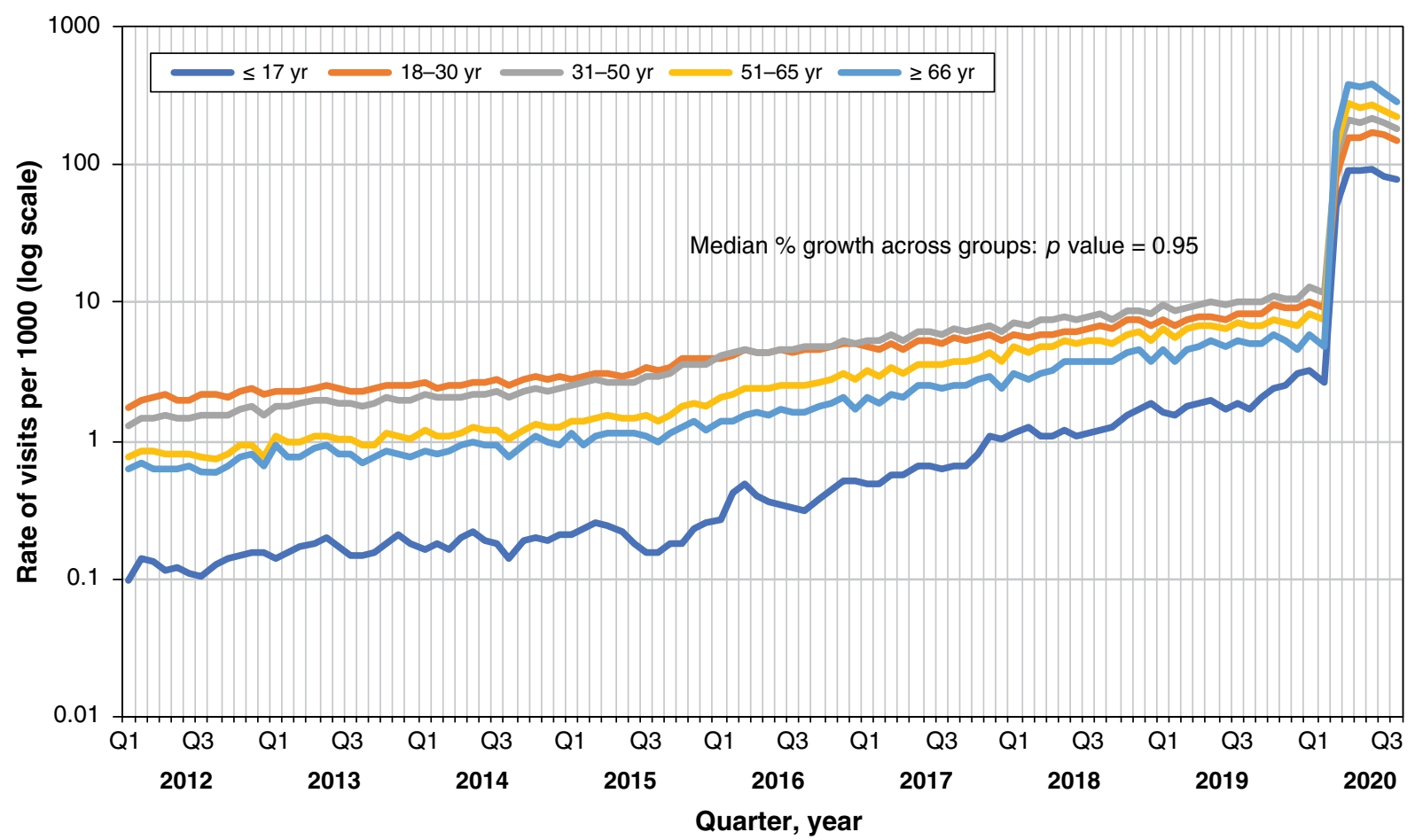

Figure 1: Rate of virtual visits per 1000 eligible Ontario patients by age group, 2012-2020.

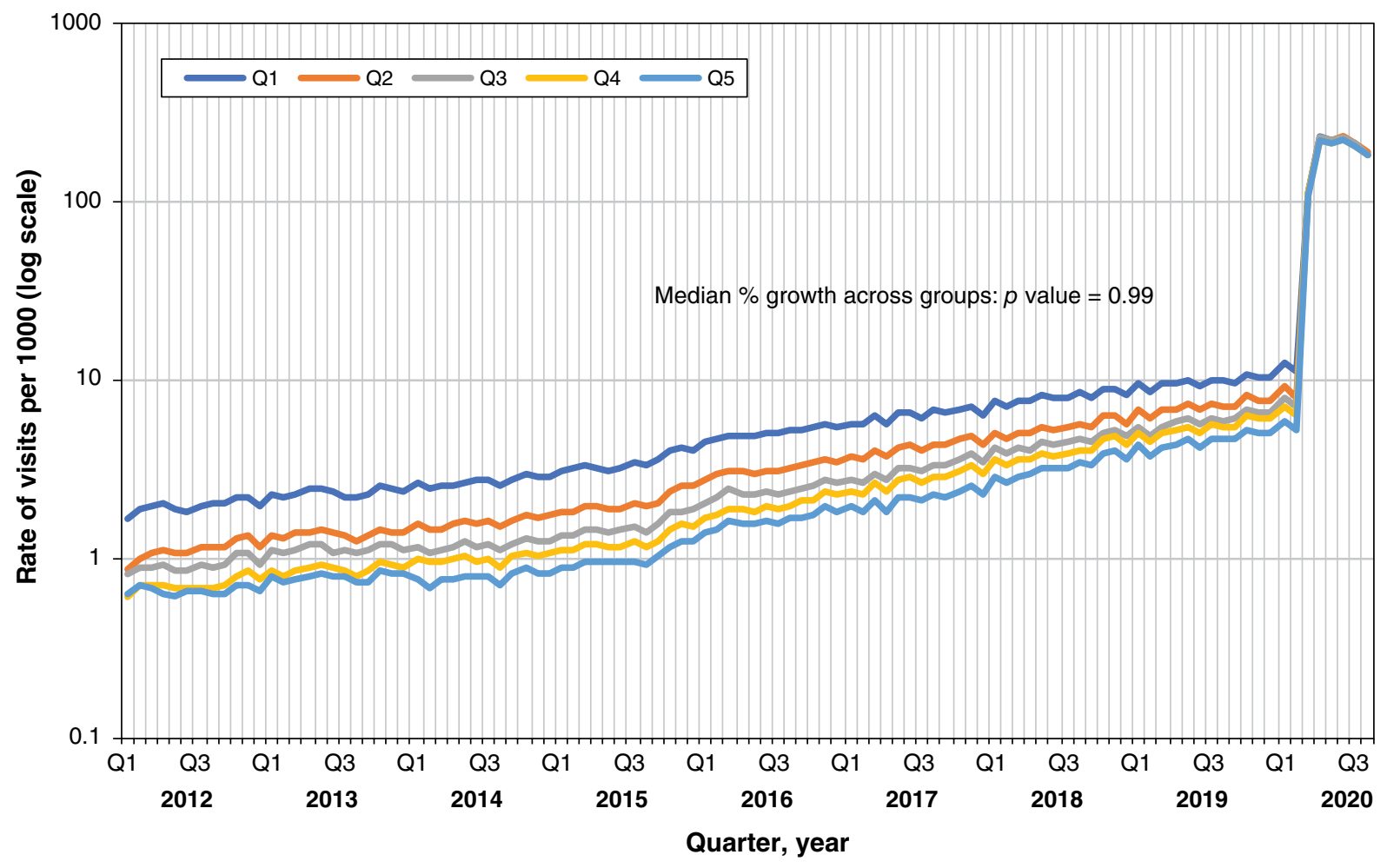

Figure 2: Rate of virtual visits per 1000 eligible Ontario patients by neighbourhood income quintile, 2012-2020. Quintile $1=$ lowest income quintile, quintile $5=$ highest income quintile. 
A

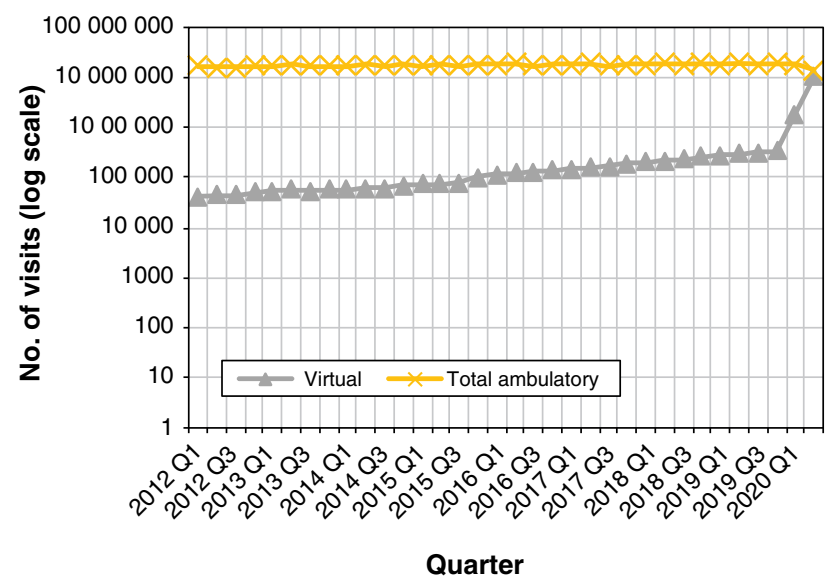

C

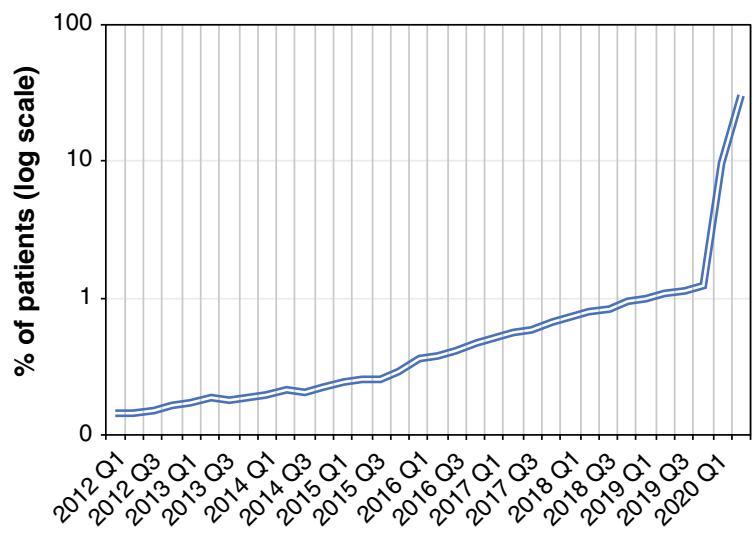

Quarter
B

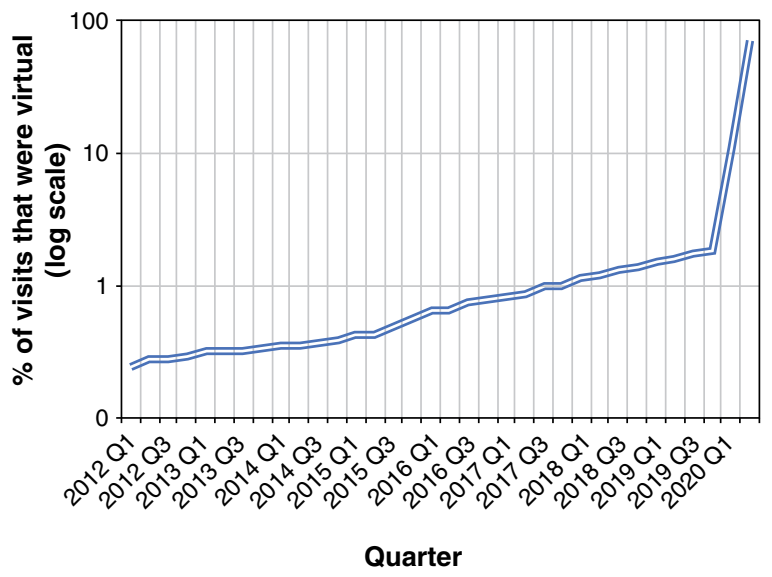

D

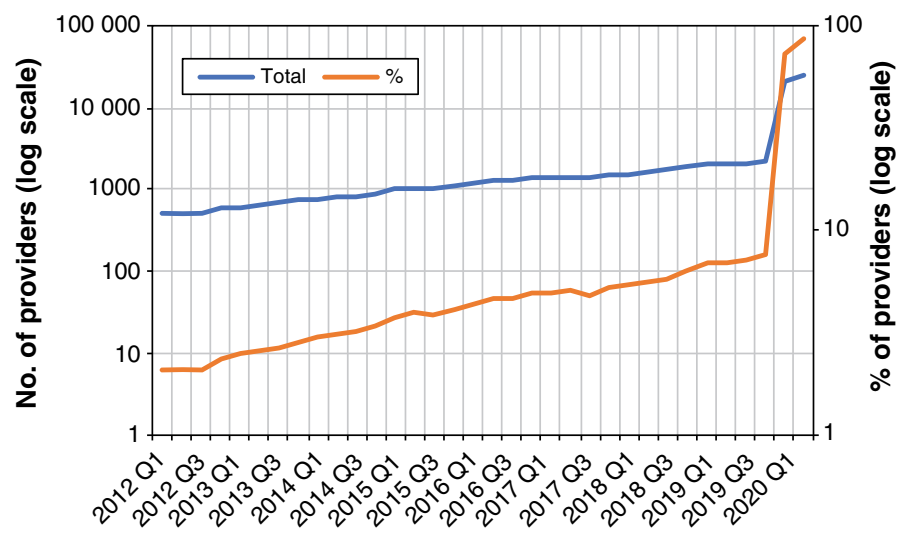

Quarter

Figure 3: (A) Number of virtual visits compared to total ambulatory visits by quarter, 2012-2020. (B) Proportion of ambulatory visits that were virtual by quarter, 2012-2020. (C) Proportion of eligible Ontario patients with virtual visits by quarter, 2012-2020. (D) Number and proportion of providers who provided virtual care by quarter, 2012-2020.

of 2020. The baseline physician characteristics among low versus high users of virtual care are shown in Appendix 1, Supplemental Table S3. Appendix 1, Supplemental Table S4 lists the physician specialties that did and did not provide virtual care services in 2020, with the distribution of virtual visits for each specialty.

The proportion of Ontario residents who had 1 or more virtual visits per quarter initially increased gradually, from $0.2 \%$ in 2012 to $1.3 \%$ in 2019 , but then increased rapidly, to $29.2 \%$ in the second quarter of 2020 (Figure 3C). Uptake of virtual care by physicians was gradual during the prepandemic period, then increased rapidly, with $85.9 \%$ of providers delivering virtual care in the second quarter of 2020 (Figure 3D). Video visit use increased by $40 \%$ between 2019 and 2020; however, overall video visit use accounted for only $8.8 \%$ of all virtual visits during 2020 . Most virtual visits
(91.2\%) during 2020 were telephone visits that were billed with the use of the new temporary billing codes.

Figure 4 shows the rate of virtual care use among patients with ambulatory-care-sensitive conditions and mental illness. The rates of virtual care use increased similarly across all conditions. In the second quarter of 2020, patients with mental health conditions had the highest rate of virtual care visits per 100 patients per quarter (237), followed by those with heart failure (181), chronic obstructive pulmonary disease (166), angina (163), diabetes (141), hypertension (124) and asthma (95).

\section{Interpretation}

In this repeated cross-sectional study, we found a large increase in virtual visits in Ontario coinciding with the COVID-19 pandemic. In contrast to expectations, we 


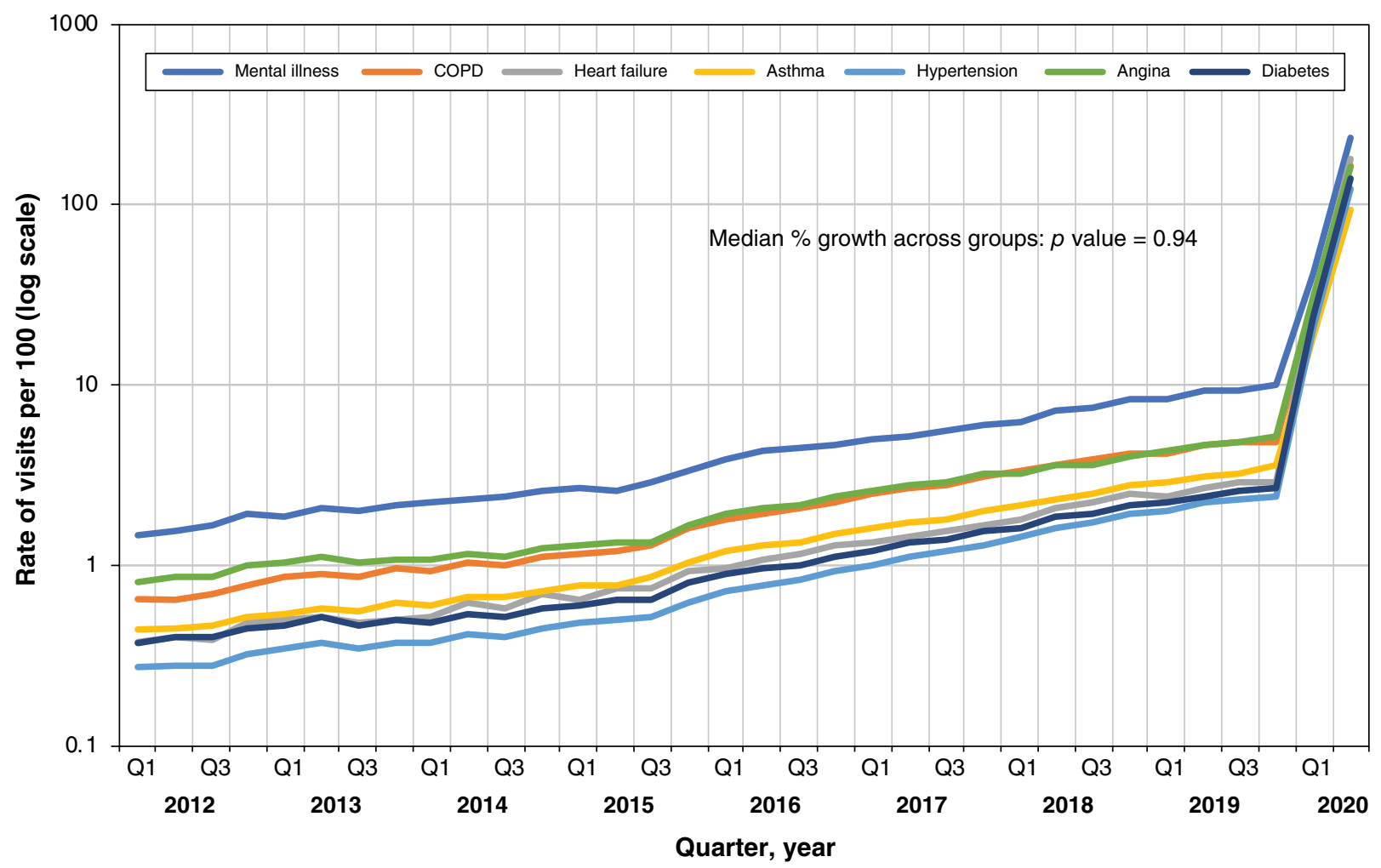

Figure 4: Rate of virtual visits per 100 eligible Ontario patients by chronic disease, 2012-2020. Note: COPD = chronic obstructive pulmonary disease.

observed similar growth in virtual care use among younger and older patients, and among patients residing in wealthier and poorer neighbourhoods. Although there was a modest increase in video visits, most virtual care was delivered through telephone calls, enabled by new billing codes introduced early in the pandemic.

Before the pandemic, only a small proportion of physicians were providing virtual care. After the arrival of COVID-19, most Ontario physicians were providing virtual care, and the majority of Ontario residents received at least 1 virtual visit during the first 6 months of 2020. Overall, the results suggest widespread physician and patient adoption of virtual care in response to the pandemic, and older age and lower income do not seem to be barriers to receiving virtual care.

Our study supports some of the early research showing increased uptake of virtual care during the pandemic. Alexander and colleagues ${ }^{10}$ reported that virtual care use increased from $4 \%$ before the pandemic to $35 \%$ during the pandemic in US primary care practices, and Baum and colleagues ${ }^{9}$ noted that the use of virtual care in Veterans Affairs facilities doubled during the pandemic. Similarly, Mehrotra and colleagues ${ }^{19}$ noted increases in virtual care use during the pandemic in a commercially insured US population. These studies were limited to particular subsets of the US population and thus had limited ability to provide a comprehensive population-level evaluation of virtual care use.
Our results add to the existing literature, for a few reasons. Our study was population-based, encompassing all 14.6 million residents of Ontario, which is demographically, culturally and geographically diverse. Universal coverage of physician and hospital services allowed us to capture data on virtual care use from several groups, including vulnerable populations, that otherwise would be excluded from studies of commercial insurance. Finally, we captured virtual care use not only in primary care but in specialty care as well.

Although video visits increased modestly during JanuaryJune 2020, the majority of virtual care was provided through telephone visits. Our results suggest the crucial role of government modification of payment rules in enabling the transition to virtual care in response to the COVID-19 pandemic.

Telephone visits are easy to use and inexpensive, with technology that is commonly available. Potential advantages of telephone over video visits include no requirement for broadband (particularly in rural and northern parts of the province) and ability to scale quickly. ${ }^{20}$ The observed higher use of telephone visits over video visits is in keeping with the literature,,$^{2,21}$ and, in health care systems that rely on large virtual care programs, telephone calls and asynchronous messaging still represent the bulk of the virtual care provided. ${ }^{22}$

The finding that the uptake of virtual visits during the early months of COVID-19 was similar among lower- and higherincome patients is important. There is concern that because 
lower-income patients may be less likely to have smartphones and broadband access, virtual care may worsen income disparities. ${ }^{23}$ Our finding suggests otherwise. Likewise, our finding of similar adoption of virtual care for older and younger patients seems to dispute the assumption that older patients are less comfortable with technology and therefore would be less likely to use virtual care. Mortality due to COVID-19 increases significantly with age; thus, older adults are at the highest risk from infection and are therefore precisely the population who would be expected to benefit the most from avoiding in-person visits when a reasonable substitute (virtual visits) is available. Our data suggest that barriers to virtual care adoption among this group may not be as important as initially thought. ${ }^{24}$

Our finding that virtual care use increased similarly for all health conditions during the COVID-19 pandemic is also noteworthy. Prior research suggesting that virtual care may be particularly effective for mental health issues may explain the higher use of virtual care in this population. Virtual care has also been shown to improve care for patients with heart failure, ${ }^{25}$ chronic obstructive pulmonary disease, ${ }^{26,27}$ coronary artery disease ${ }^{28}$ and diabetes. ${ }^{29}$ Further study is required to understand which diseases and conditions are most amenable to virtual care, the optimal dosing of virtual care (frequency of visits, duration) and the content of visits (e.g., symptom assessment, medication reconciliation). Finally, it is critical to measure the quality of the virtual care visit, defined by the Institute for Healthcare Improvement's Quadruple Aim (patient experience, population health, provider experience and cost)..$^{30}$ Virtual care should not be treated differently from other elements of the health care experience and should lead to a positive patient and provider experience, improved health outcomes and lowered costs. ${ }^{31}$

\section{Limitations}

We lack the clinical indications for the virtual visits analyzed and the requisite detail to assess the content or quality of care of the visits. Likewise, further study is needed to assess the quality of virtual visits relative to in-person visits, as well as impact on health care costs and patient outcomes. Our reliance on administrative billing codes precludes us from knowing precisely what type of video platform (e.g., unsecure videoconference such as FaceTime) was used by physicians who billed the temporary codes. Finally, our study was conducted in a single-payer health system, and the results may not be generalizable to other settings, particularly health care systems that do not have universal coverage of physician and hospital services or are multipayer.

\section{Conclusion}

Ontario responded to the COVID-19 pandemic with changes that facilitated widespread adoption of virtual visits as a substitute for in-person care. Importantly, use of virtual care increased to similar degrees across the entire population, including traditionally vulnerable subgroups. Future research should focus on how to assess the quality of virtual care and identification of diseases and patient subgroups for which virtual care may be less effective than in-person visits.

\section{References}

1. Simpson AT, Doarn C, Garber S. Interagency cooperation in the twilight of the great society: telemedicine, NASA, and the Papago nation. 7 Policy Hist 2020;32:25-51.

2. Stamenova V, Agarwal P, Kelley L, et al. Uptake and patient and provider communication modality preferences of virtual visits in primary care: a retrospective cohort study in Canada. BM7 Open 2020;10:e037064.

3. Roland M. General practice by smartphone. BM7 2019;366:14713.

4. 2019 CMA annual report. Ottawa: Canadian Medical Association. Available: www.cma.ca/sites/default/files/pdf/AGM/2019-annual-report-w-cover-v2-e.pdf (accessed 2020 Oct. 20)

5. Shaw J, Jamieson T, Agarwal P, et al. Virtual care policy recommendations for patient-centred primary care: findings of a consensus policy dialogue using a nominal group technique. 7 Telemed Telecare 2018;24:608-15.

6. CMA Health Summit: virtual care in Canada [discussion paper]. Ottawa: Canadian Medical Association. Available: www.cma.ca/sites/default/files/pdf/News/ Virtual_Care_discussionpaper_v2EN.pdf (accessed 2020 Oct. 21).

7. Sim I. Mobile devices and health. N Engl F Med 2019;381:956-68.

8. Bhatia RS, Shojania KG, Levinson W. Cost of contact: redesigning healthcare in the age of COVID. BM7 Qual Saf 2020 Aug. 6 [Epub ahead of print]. doi: 10.1136/bmjqs-2020-011624.

9. Baum A, Kaboli PJ, Schwartz MD. Reduced in-person and increased telehealth outpatient visits during the COVID-19 pandemic. Ann Intern Med 2020 Aug. 10 [Epub ahead of print]. doi: 10.7326/M20-3026.

10. Alexander GC, Tajanlangit M, Heyward J, et al. Use and content of primary care office-based vs telemedicine care visits during the COVID-19 pandemic in the US. 7AMA Netw Open 2020;3:e2021476.

11. Postal Code ${ }^{\mathrm{OM}}$ Conversion File (PCCF), reference guide. Ottawa: Statistics Canada; 2017. Available: https://www150.statcan.gc.ca/n1/pub/92-154-g/92 -154-g2017001-eng.htm (accessed 2021 Feb. 2).

12. ICES Data Dictionary. Available: https://datadictionary.ices.on.ca/ (accessed 2020 Sept. 9).

13. Hux JE, Ivis F, Flintoft V, et al. Diabetes in Ontario: determination of prevalence and incidence using a validated administrative data algorithm. Diabetes Care 2002;25:512-6.

14. Tu K, Campbell NR, Chen ZL, et al. Accuracy of administrative databases in identifying patients with hypertension. Open Med 2007;1:e18-26.

15. Crighton EJ, Feng J, Gershon A, et al. A spatial analysis of asthma prevalence in Ontario. Can 7 Public Health 2012;103:e384-9.

16. Di Giuseppe G, Chu A, Tu JV, et al. Incidence of heart failure among immigrants to Ontario, Canada: a CANHEART immigrant study. 7 Card Fail 2019;25:425-35.

17. Kurdyak P, Lin E, Green D, et al. Validation of a population-based algorithm to detect chronic psychotic illness. Can 7 Psychiatry 2015;60:362-8.

18. Kralj B. Measuring rurality - RIO2008_BASIC: methodology and results. Toronto: Ontario Medical Association; 2009. Available: https://content.oma. org//wp-content/uploads/2008rio-fulltechnicalpaper.pdf (accessed 2020 Sept. 9).

19. Mehrotra A, Chernew M, Linetsky D, et al. The impact of the COVID-19 pandemic on outpatient visits: a rebound emerges [blog]. New York: The Commonwealth Fund; 2020 May 19. Available: www.commonwealthfund.org/ publications/2020/apr/impact-covid-19-outpatient-visits (accessed 2020 Oct. 20).

20. Jaklevic MC. Telephone visits surge during the pandemic, but will they last? 7AMA 2020 Oct. 7 [Epub ahead of print]. doi: 10.1001/jama.2020.17201.

21. Wootton R. Twenty years of telemedicine in chronic disease management: an evidence synthesis. $\mathcal{F}$ Telemed Telecare 2012;18:211-20.

22. Pearl R. Kaiser Permanente Northern California: current experiences with internet, mobile, and video technologies. Health Aff (Millwood) 2014;33:251-7.

23. Eberly LA, Kallan MJ, Julien HM, et al. Patient characteristics associated with telemedicine access for primary and specialty ambulatory care during the COVID-19 pandemic. FAMA Netw Open 2020;3:e2031640.

24. Kruse C, Fohn J, Wilson N, et al. Utilization barriers and medical outcomes commensurate with the use of telehealth among older adults: systematic review. 7MIR Med Inform 2020;8:e20359.

25. Gensini GF, Alderighi C, Rasoini R, et al. Value of telemonitoring and telemedicine in heart failure management. Card Fail Rev 2017;3:116-21.

26. McLean S, Nurmatov U, Liu JLY, et al. Telehealthcare for chronic obstructive pulmonary disease: Cochrane Review and meta-analysis. Br 7 Gen Pract 2012; 62:e739-49.

27. Lundell S, Holmner $\AA$, Rehn B, et al. Telehealthcare in COPD: a systematic review and meta-analysis on physical outcomes and dyspnea. Respir Med 2015; 109:11-26.

28. Ivers NM, Schwalm JD, Bouck Z, et al. Interventions supporting long term adherence and decreasing cardiovascular events after myocardial infarction (ISLAND): pragmatic randomised controlled trial. BM7 2020;369:m1731.

29. McDonnell ME. Telemedicine in complex diabetes management. Curr Diab Rep 2018; 18:42

30. Feeley D. The Triple Aim or the Quadruple Aim? Four points to help set your strategy [blog]. Available: www.ihi.org/communities/blogs/the-triple-aim-or-the -quadruple-aim-four-points-to-help-set-your-strategy (accessed 2021 Feb. 2)

31. Sikka R, Morath JM, Leape L. The Quadruple Aim: care, health, cost and meaning in work. BM7 Qual Saf 2015;24:608-10. 
Affiliations: Women's College Hospital Institute for Health Systems Solutions and Virtual Care (Bhatia, Chu, Tadrous, Stamenova), Women's College Hospital; ICES Central (Bhatia, Pang); Leslie Dan Faculty of Pharmacy (Tadrous), University of Toronto; Division of General Internal Medicine and Geriatrics (Cram), Sinai Health System and University Health Network; Department of Medicine (Cram), University of Toronto, Toronto, Ont.

Contributors: Sacha Bhatia conceived of the study. Cherry Chu and Andrea Pang analyzed the data. Sacha Bhatia, Vess Stamenova and Peter Cram interpreted the data. Sacha Bhatia drafted the manuscript. All of the authors contributed to the study design, revised the manuscript critically for important intellectual content, approved the final version to be published and agreed to be accountable for all aspects of the work.

Funding: This study was supported by ICES, which is funded by an annual grant from the Ontario Ministry of Health and Long-Term Care. This work was also supported by grant R01AG058878 to Peter Cram from the US National Institute on Aging.

Data sharing: The data set from this study is held securely in coded form at ICES. Although data-sharing agreements prohibit ICES from making the data set publicly available, access may be granted to those who meet prespecified criteria for confidential access, available at http://www.ices. on.ca/DAS. The full data set creation plan and underlying analytic code are available from the authors on request, with the understanding that the computer programs may rely on coding templates or macros that are unique to ICES and are therefore either inaccessible or may require modification.

Disclaimer: This study was supported by ICES, which is funded by an annual grant from the Ontario Ministry of Health and Long-Term Care (MOHLTC). Parts of this material are based on data and information compiled and provided by the MOHLTC and the Canadian Institute for Health Information. The analyses, conclusions, opinions and statements expressed herein are solely those of the authors and do not reflect those of the funding or data sources; no endorsement is intended or should be inferred.

Content licence: This is an Open Access article distributed in accordance with the terms of the Creative Commons Attribution (CC BY-NCND 4.0) licence, which permits use, distribution and reproduction in any medium, provided that the original publication is properly cited, the use is noncommercial (i.e., research or educational use), and no modifications or adaptations are made. See: https://creativecommons.org/licenses/ by-nc-nd/4.0/.

Supplemental information: For reviewer comments and the original submission of this manuscript, please see www.cmajopen.ca/content/9/1/ E107/suppl/DC1. 